This item was submitted to Loughborough's Research Repository by the author.

Items in Figshare are protected by copyright, with all rights reserved, unless otherwise indicated.

\title{
Bus driver fatigue: a qualitative study of drivers in London
}

PLEASE CITE THE PUBLISHED VERSION

https://doi.org/10.1016/j.apergo.2020.103309

PUBLISHER

Elsevier BV

VERSION

AM (Accepted Manuscript)

PUBLISHER STATEMENT

This paper was accepted for publication in the journal Applied Ergonomics and the definitive published version is available at https://doi.org/10.1016/j.apergo.2020.103309.

\section{LICENCE}

CC BY-NC-ND 4.0

\section{REPOSITORY RECORD}

Maynard, Sally, Ashleigh Filtness, Karl Miller, and Fran Pilkington-Cheney. 2020. "Bus Driver Fatigue: A Qualitative Study of Drivers in London”. Loughborough University. https://hdl.handle.net/2134/13326401.v1. 


\title{
Bus Driver Fatigue: A qualitative study of drivers in London
}

\author{
Sally Maynard, Ashleigh Filtness, Karl Miller, Fran Pilkington-Cheney
}

Transport Safety Research Centre, School of Design and Creative Arts, Loughborough University, Loughborough LE11 3TU

\begin{abstract}
The research represents one of the first major qualitative studies to subjectively investigate fatigue amongst city bus drivers. Funded by TfL (grant no. 94050), the study considers the perspectives of both the bus drivers themselves and their managers. It explores opinion specifically in relation to how fatigue influences or is influenced by bus driving, and how this is managed. Data was collected from focus groups with bus drivers ( $n=65$ drivers) and one-to-one interviews with bus driver managers $(n=11)$. Fatigue was seen to be a problem, with all drivers having experienced for themselves the feeling of sleepiness whilst driving the bus, or knowing about it in others. The managers agreed that fatigue amongst drivers is evident, seeing it particularly as a contributor to serious incidents. Fatigue and sleepiness are not discussed between drivers as a general rule and they do not feel comfortable reporting instances of fatigue for fear of being disciplined. Managers would encourage drivers to report these issues and therefore hoped to create a more open culture. Factors identified as contributors to fatigue included: working overtime, having insufficient sleep, spending time with family, other non-work commitments, shift schedules, and work pressure.
\end{abstract}

Keywords: fatigue, sleepiness, bus drivers, focus groups, interviews, road safety

\section{Introduction}

Driver fatigue is a significant issue which has been estimated to contribute to $15 \%-30 \%$ of road traffic crashes (Connor et al., 2002; Horne \& Reyner, 1995; Phillip et al., 2014) and is a globally known causation factor contributing to crashes, injuries, and deaths in various transportation operations (Williamson et al., 2011; Bioulac et al., 2017; Zhang, Yan, Wu, \& Qiu, 2014). It has been shown that driving while fatigued results in decrements in performance for both simple and complex tasks, impaired attention, slowed reaction times, and loss of conscious awareness while behind the wheel (see Williamson et al., 2011 for review).

The very limited available research with city bus drivers has indicated that sleepiness can be a major issue, with approximately $45 \%$ of Swedish bus drivers having to fight against sleepiness at least 2-4 times each month whilst driving the bus, and $19 \%$ of drivers fighting to stay awake at least 2-3 times per week (Anund et al., 2016). Similar findings have been reported for London city bus drivers, with approximately $36 \%$ reporting having to fight sleepiness while driving the bus at least 2-4 times per month, and $21 \%$ of drivers fighting to stay awake at least 2-3 times a week (Miller et al., 2020). A previous study conducted in Edinburgh found that $20 \%$ of bus drivers reported excessive daytime sleepiness, citing scores of more than 10 on the Epworth Sleepiness Scale (ESS), a subjective scale designed to assess trait daytime sleepiness in which scores of 10 or more are associated with excessive daytime sleepiness. $12 \%$ of drivers were also found to have fallen asleep at the wheel at least once per month, $7 \%$ had had been involved in an accident and $8 \%$ of drivers had experienced a near miss due to sleepiness while driving (Vennelle et al., 2010). Most recently, Miyama et al. (2020) carried out a questionnaire survey of bus drivers in Japan $(n=301)$ and found that $44.5 \%$ of respondents reported near-miss incidents in the month preceding the survey and $11.7 \%$ had experienced collisions once or twice. When asked about the main causes of major accidents, $64.1 \%$ cited heavy traffic, $63.0 \%$ cited poor working/break conditions, $50.0 \%$ cited lack of safety education 
and $32.8 \%$ cited irresponsible behaviour by drivers. Notably, around $37 \%$ of participating drivers reported that they were reluctant to alert the company when feeling sick.

Although there is evidence that city bus drivers experience sleepiness while driving, the great majority of the previous work related to sleepiness in city bus drivers is quantitative in nature. There is therefore a lack of subjective research exploring bus driver opinion relating to their experience of bus driving and how fatigue influences or is influenced by this, and how this is managed. This means there is a considerable gap in the knowledge about bus driver fatigue because, up to now, those who are actually experiencing it have not had the opportunity to explain their direct views and thoughts. Qualitative research is complementary to quantitative studies and, in this case, can provide more indepth explanations from the drivers themselves as opposed to the interpretation of the researchers who do not have the lived experience of working as a fatigued bus driver. Without understanding the reality of daily operations, the design of any fatigue risk management policy may be limited, since real life practice may differ from what policy or procedure suggests. An example of where this was shown to be the case for train drivers can be seen in Filtness \& Naweed (2017).

A notable exception in the area of qualitative research is by Biggs et al. (2009) who identified the following key themes in relation to bus driver fatigue: level of support from management; policing of ticketing and related issues; interaction with passengers; cabin ergonomics; tight route schedules; turn-around and shift irregularity; extended shift cycles; interactions with other road users; and drivers' extended commute times. This 2009 study involved just five focus groups with metropolitan bus drivers ( $\mathrm{n}=$ not specified) and other jobs involving a bus operation component (e.g. trainers, assessors and customer service consultants) in Australia. It is notable that the research being described in this paper focuses on a greater number of drivers working in a larger bus network than previous qualitative studies.

The current work was funded by TfL (grant no. 94050) and addresses this lack of research by talking directly to bus drivers in focus groups and separately interviewing their managers. It takes place in one major city and in one of the biggest bus networks in the world with 700 routes and 2.5 million passengers and involves a greater number of focus groups than Biggs et al. (2009). In addition, it includes only bus driver participants in the focus groups, with other job roles considered separately with interviews. It represents further investigation to "refine and quantify the effects of driver fatigue" which is recommended by Biggs et al. (2009).

For the purpose of this research, driver fatigue is defined as: "a psychological and/or physical impairment which has the potential to reduce optimal performance. Fatigue is considered to be multifaceted, encompassing pressures from both endemic sleepiness relating to the body's homeostatic and circadian pressures, and task related fatigue". The researchers recognise that there is a difference between fatigue and sleepiness and were interested in both concepts throughout the research. For a detailed discussion of fatigue and sleepiness see Pilkington-Cheney et al. (2020b). At the beginning of each focus group the concept of 'fatigue' was discussed in order to be sure that participants were aware of its meaning, but at the same time researchers attempted not to restrict their thoughts by enforcing constricting definitions. Relevant for this study is the fact that, in occupational settings, sleepiness and fatigue are often used interchangeably. Within the transport industry the term fatigue is used frequently and is therefore predominately employed here. Within the current work a single investigation is undertaken to investigate fatigue, while recognising that some aspects relate directly to sleepiness. This broad usage of the term fatigue to include sleepiness was employed during the data gathering. 
The focus groups and interviews discussed here were intended to address the following research questions:

- How does the experience of fatigue manifest for London bus drivers?

- What are the key contributors to fatigue according to London bus drivers?

- Do London bus drivers report fatigue?

- What are the differences in opinion between London bus drivers and their managers in relation to fatigue?

\section{Methodology}

Two research tasks were undertaken: 1 . Focus groups with bus drivers and 2. Interviews with bus driver managers. The research was funded by Transport for London (TfL), an organisation which manages one of the largest bus networks in the world, with around 9,300 vehicles in the fleet. Approximately 26,000 employees drive TfL's buses; it is important to note here that the majority of bus services in London are run by ten private operators awarded a competitive contract by TfL (Transport for London, 2019) so bus drivers and managers are not direct employees of TfL. It was therefore crucial that all ten operators had some input into the research in order to ensure it was as comprehensive and inclusive as possible. It should also be noted that the appearance to customers is of one London bus service, but the driver experience is that of working for different companies.

Most of the operators run a mixture of shifts and five of the operators work 24 hours per day, seven days per week. It is therefore difficult to be exact on the daily number of hours worked by drivers. In addition, at all ten of the TfL operators, regulations are in place to specify a required maximum shift and minimum break duration for drivers. Table 1 shows the driving hours and regulations as specified by the Vehicle Operator Services Agency [V.O.S.A] (2015), for Great Britain, which apply to London bus drivers (along with most urban bus drivers). These regulations necessarily limit somewhat the extent to which drivers are permitted to work.

\begin{tabular}{|l|l|}
\hline Daily driving limit & 10 hours \\
\hline Maximum continuous driving & $\begin{array}{l}5 \frac{1}{2} \text { hours, then 30-minute } \\
\text { break }\end{array}$ \\
\hline Maximum spreadover & 16 hours \\
\hline Minimum daily rest & 10 hours (can be reduced to \\
& $8 \frac{1}{2}$ hours up to 3 times per \\
& week) \\
\hline Fortnightly limits & Must include 1 rest day \\
\hline
\end{tabular}

Table 1: Driving hours and regulations for London bus drivers

In accordance with Loughborough University's code of practice for research, ethical clearance was sought and gained via the relevant procedures.

\subsection{Focus groups with bus drivers}

Focus groups were held to explore the experiences of fatigue as reported by London bus drivers; these were semi-structured in that there was a guide covering major questions but the sequence of the questions and the level of probing for information varied in order to allow the discussion to develop organically. The guide was intended to ensure overall consistency between groups and the questions were as open-ended as possible and participants were encouraged to talk to each other as well as to the researchers, meaning that a greater amount of information could be elicited. The 
participating drivers were encouraged to treat the experience as an informal discussion about their experiences of fatigue and how it is managed at work, and to express their honest opinions. The topics discussed and example questions can be seen in Table 2 . The focus group guide was informed by issues identified during a review of relevant literature and the prior knowledge held within the research team. The project's definition of fatigue was explained at the beginning of each group in order to ensure consistent understanding amongst the participants.

At each of the different private operators, one group of 6-8 drivers were invited to attend the discussion. They were selected by the operator at which they were employed with a balanced mix of those who had been employed long-term together with those having been driving for a shorter period (but for at least 6 months). Participation was voluntary and drivers were remunerated at their normal pay rate for the time away from work. Basic demographic data was collected from the participants in a short questionnaire including that relating to shifts worked - this proved to vary widely. All participants gave their informed consent.

The data collection took place in October and November 2018 at a London bus depot or garage nominated by the relevant operator. The discussion occurred in a private room where it could not be overheard by anyone outside and no managers were present. Focus groups were facilitated by two researchers; for consistency the first author was present at all data collection sessions. The discussions lasted between 60 and 90 minutes and were audio recorded with the permission of all participants.

\begin{tabular}{|l|l|}
\hline Topic & Example question \\
\hline Introduction & What made you decide to become a bus driver? \\
\hline Experience of fatigue & $\begin{array}{l}\text { Could you describe a situation where you or anyone you know } \\
\text { has suffered from driver fatigue during work? }\end{array}$ \\
\hline $\begin{array}{l}\text { Causes and consequences of } \\
\text { fatigue }\end{array}$ & $\begin{array}{l}\text { In what way do you think fatigue/sleepiness influences safety } \\
\text { at work? }\end{array}$ \\
\hline Strategies to manage fatigue & What do you do if you feel fatigued/sleepy at work? \\
\hline Workplace culture & $\begin{array}{l}\text { Could you describe how you would communicate with your } \\
\text { employer or supervisor if you were fatigued/sleepy at work? }\end{array}$ \\
\hline $\begin{array}{l}\text { Sleep and fatigue outside } \\
\text { work }\end{array}$ & $\begin{array}{l}\text { What factors influence how much you would sleep when not at } \\
\text { work? }\end{array}$ \\
\hline Shift patterns and overtime & $\begin{array}{l}\text { Are shift patterns fixed or can you/do you swap shifts? How } \\
\text { does this influence fatigue? }\end{array}$ \\
\hline
\end{tabular}

Table 2: Overview of focus group topics

\subsection{Manager interviews}

Manager interviews followed on from the bus driver focus groups with data collection taking place in November 2018. Suitable participants were identified by a contact at each operator and were defined as 'A person who would respond if a driver reports feeling tired, or if a driver has an incident appearing to be caused by fatigue/sleepiness'. The participants were therefore a selection of immediate driver supervisors and higher-level managers within the bus companies.

A manager interview question guide was produced in order to ensure each interview followed a similar format and details of the topics and examples of the questions can be found in Table 3. The guide arose from the findings of a wider literature review, the bus driver focus groups, and the prior 
knowledge and experience within the research team. The interviews explored the understanding of fatigue and sleepiness in the industry, its effects, and how it is managed. The project's definition of fatigue was explained at the beginning of each interview in order to ensure consistent understanding and all participants gave informed consent.

The interviews lasted 30-45 minutes and were audio recorded with the permission of the participants. They were all conducted by telephone and by the same researcher.

\begin{tabular}{|l|l|}
\hline Topic & Example question \\
\hline Introduction & What brought you to your current role? What does it entail? \\
\hline $\begin{array}{l}\text { The prevalence of } \\
\text { fatigue/sleepiness among bus } \\
\text { drivers }\end{array}$ & $\begin{array}{l}\text { Have you ever experienced a driver reporting suffering from } \\
\text { fatigue at work? If so, what happened and what did you do } \\
\text { about it? }\end{array}$ \\
\hline $\begin{array}{l}\text { The causes and consequences of } \\
\text { fatigue/sleepiness in bus drivers }\end{array}$ & $\begin{array}{l}\text { What issues do you think cause drivers to be fatigued at } \\
\text { work? }\end{array}$ \\
\hline $\begin{array}{l}\text { The strategies used to manage } \\
\text { fatigue/sleepiness at work }\end{array}$ & $\begin{array}{l}\text { What do you believe the company is doing well/poorly in } \\
\text { the management of fatigue/sleepiness? }\end{array}$ \\
\hline Shift patterns/overtime & $\begin{array}{l}\text { Are shift patterns fixed or does the company allow drivers } \\
\text { to swap shifts? Do you think this has an effect on fatigue? }\end{array}$ \\
\hline
\end{tabular}

Table 3: Overview of manager interview topics

\subsection{Participant Demographics}

All ten of the London bus operating companies participated in bus driver discussions in some way. That is, eight focus groups with 6-8 participants were held whereas at one operator two groups of four were organised due to the lack of availability of drivers at one time. Results of the two groups were analysed together and will be considered to represent one focus group in the analysis of this work. The last operator was also unable to provide 6-8 drivers at one time and arranged three interviews with single drivers. The data from the three interviews were considered together as one focus group for the analysis of this study. The data from all of these interactions with drivers has been combined and will from this point be referred to as being from the focus groups.

The demographic data collected from the participating drivers can be seen in Table 4. Notably, thirteen participants ( $21 \%$ of those responding) had been working in the bus industry for more than 25 years, demonstrating the long service of these drivers.

\begin{tabular}{|l|l|}
\hline \multicolumn{2}{|c|}{ Age of participants (drivers) } \\
\hline $25-34$ years & $16.9 \%(n=11)$ \\
\hline $35-44$ years & $21.5 \%(n=14)$ \\
\hline $45-54$ years & $35.4 \%(n=23)$ \\
\hline $55-64$ years & $26.2 \%(n=17)$ \\
\hline Total (including three interviewees) & $100 \%(n=65)$ \\
\hline \multicolumn{2}{|c|}{ Gender of participants (drivers) } \\
\hline Female Participants' service as a driver \\
\hline Male & $20 \%(n=13)$ \\
\hline \multicolumn{2}{|c|}{$80 \%(n=52)$} \\
\hline $\begin{array}{l}\text { Average period spent working as a } \\
\text { bus driver }\end{array}$ & 13.7 years (SD 10.6; \\
Range 0.5 - 41)
\end{tabular}




\begin{tabular}{|l|l|}
\hline $\begin{array}{l}\text { Average period spent working for } \\
\text { current employer }\end{array}$ & $\begin{array}{l}8.1 \text { years (SD 8.1; } \\
\text { Range } 0.5-41)\end{array}$ \\
\hline
\end{tabular}

Table 4: Demographic data provided by participating drivers

A variety of different shift schedules were being worked with the majority of drivers (89\%) working early shifts; in many cases this was one kind of shift amongst a range, particularly where drivers were working rotating shift patterns. The shifts worked were self-selected without specific times due to variations between the operators - more details can be seen in Table 5 .

\begin{tabular}{|l|r|}
\hline Type of shift & \multicolumn{1}{|l|}{ Number* } \\
\hline Early & 58 \\
\hline Middle & 39 \\
\hline Late & 32 \\
\hline Night & 7 \\
\hline Total & 136 \\
\hline
\end{tabular}

Table 5: Types of shift driven

* Respondents were able to select more than one option

All ten of the London bus operating companies nominated managers suitable to be interviewed in relation to fatigue management. In total, 11 one-to-one telephone interviews were conducted by the lead author (two individuals from one operator; one at each of the remaining nine operators). Ten of the interviewees had previously worked as a bus driver before becoming a manager and two were female with the remaining nine being male. The managers had been working in a management role for their current employer for an average of 11 years (SD 10.3; Range 1-32); for male managers only it was 13.1 years (SD 10.3; Range 2 - 32) and for female managers it was 1.5 years (SD 0.7; Range 1 - 2). The participants had taken on a variety of roles including controller and supervisor. Some had also moved between different bus companies or had worked in similar organisations such as coach operators. They were not required to provide details of their age.

\subsection{Data analysis}

The recordings of the focus groups (682 minutes total) and manager interviews (395 minutes total) were transcribed verbatim, de-identified and analysed by a team of three researchers using thematic analysis (Braun \&Clarke, 2006) via NVivo (ver. 12.0) software. The three researchers had each attended at least one of the focus groups. The dialogue from each focus group was coded in two stages of qualitative thematic data analysis: (1) open coding and (2) axial coding (Saldana, 2009). Given the size and richness of the dataset, these two stages of analysis created a robust process, ensuring all data were captured and considered during analysis.

The first stage (open coding) identified units of information that addressed the research aims. This was a bottom-up approach to obtain first impressions from the transcripts and was led by the opinions and narrative of the participants. During this stage, two of the three researchers in the team read through the data and started to create tentative labels for chunks of data that summarised the ideas arising from the data. The two researchers separately coded the transcript from the first completed focus group in this way and then, to avoid bias, met to compare their analysis and subsequently to refine and agree their final set of codes.

In the second stage (axial coding), relationships were identified among the open codes; these resonated with pre-established constructs derived from fatigue risk management theory and the professional driving context and the core themes were subsequently identified. This axial coding was a critical step for relating categories and concepts with one another and refining the categories. This 
resulted in an agreed set of 14 codes (or nodes as they are described in NVivo); these were rationalised by comparing and contrasting the nodes derived by the two researchers during their initial analysis and ensuring that all of the topics raised were covered by the new codified group. The 14 codes (or themes) were then used to analyse the remainder of the focus groups - each discussion was analysed by the member of the research team who had attended all of the focus groups.

In order to contextualise the focus group data and facilitate comparison between the drivers and the managers, the same themes as those identified from the focus groups were utilised in the subsequent analysis of the manager interviews. The consistency in use of themes between the two work roles ensured a common context. As with the focus group analysis, one interview was independently coded by the two researchers in order to ensure that the pre-established codes were appropriate for the interview data. The two coders were the researcher who conducted all of the interviews and the third member of the research team; they reviewed and discussed their results together. As with the focus groups, the themes derived by the two researchers during their initial analysis were rationalised and compared to ensure all of the topics raised were covered. The researcher who conducted the interviews undertook the analysis of the remainder of the interviews; nine of the 14 themes evident in the driver analysis were subsequently identified.

The themes which were judged to be the most significant during the focus groups and the interviews will now be discussed for the purposes of comparison and contrast.

\section{Results}

\subsection{Theme Identification}

For the focus groups, the thematic analysis resulted in a total of 53 subcategories being independently identified by two researchers. These were then agreed and sorted jointly into 14 main categories or themes, listed in Table 6 (below) with details of the number of focus groups in which these were detected. The same themes as those identified from the focus groups were utilised in the subsequent analysis of the manager interviews. Overall, nine of these 14 key themes were identified in the manager interview data, as noted in Table 6. A subgroup of the themes which were judged to be the most significant in terms of their perceived ability to influence safety and of being more often discussed during the focus groups and the interviews will now be considered further. This represents seven of the 14 themes which have been selected due to being most universal to transport and most specific to city bus drivers. They are also the most relevant to the research questions quoted at the beginning of this article. The results pertaining to the use of fatigue countermeasures is considered in more depth elsewhere (Pilkington-Cheney et al., 2020a). 


\begin{tabular}{|l|c|c|}
\hline Theme & Focus groups & $\begin{array}{c}\text { Manager } \\
\text { interviews }\end{array}$ \\
\hline Definitions and understanding of fatigue & $\mathrm{n}=10$ & $\mathrm{n}=11$ \\
\hline Reporting fatigue & $\mathrm{n}=10$ & $\mathrm{n}=11$ \\
\hline Shift patterns (and overtime) & $\mathrm{n}=10$ & $\mathrm{n}=11$ \\
\hline Sickness and ill-health & $\mathrm{n}=10$ & $\mathrm{n}=10$ \\
\hline Stress, workload and frustration & $\mathrm{n}=10$ & $\mathrm{n}=9$ \\
\hline Work and home life & $\mathrm{n}=10$ & $\mathrm{n}=10$ \\
\hline Money & $\mathrm{n}=10$ & $\mathrm{n}=8$ \\
\hline Commuting & $\mathrm{n}=9$ & $\mathrm{n}=11$ \\
\hline Facilities & $\mathrm{n}=6$ & $\mathrm{n}=4$ \\
\hline Countermeasures & $\mathrm{n}=10$ & $\mathrm{x}$ \\
\hline Sleep & $\mathrm{n}=10$ & $\mathrm{x}$ \\
\hline Time of the day & $\mathrm{n}=10$ & $\mathrm{x}$ \\
\hline The occupation of 'bus driver' & $\mathrm{n}=10$ & $\mathrm{x}$ \\
\hline Other road users & $\mathrm{n}=10$ & $\mathrm{x}$ \\
\hline
\end{tabular}

Table 6: Themes identified during research analysis ( $n=$ number of groups/interviews in which theme was detected)

The seven themes identified for further discussion here generally fall under three higher level groupings; Figure 7 gives a pictorial representation of these, which are argued to be: attitude (of bus drivers), workplace concerns and the transition between work and home. As can be seen in the Figure, there are some overlaps of lower level themes between the groupings. The themes of 'definitions and understanding of fatigue' and 'reporting fatigue' are considered to reflect the attitudes of the participating drivers (towards fatigue) and the theme entitled 'occupation of 'bus driver" encapsulates their attitude towards the role itself as well as being intimately linked to the workplace. Other themes which pertain to the workplace are 'facilities' (which should be provided at the workplace or via the employer) and 'shifts' (fixed by the employer). The transition between work and home is encapsulated by 'commuting' (the physical transfer) and the theme of 'work life and home life' (the relationship between the two). 'Shifts' are also key here because they may often cause work to impinge on home and stop drivers living in the social role which they might prefer. It is argued that this analysis demonstrates the originality of this research in bringing to the fore, in the words of the drivers themselves, this important conflict between their home life and working life.

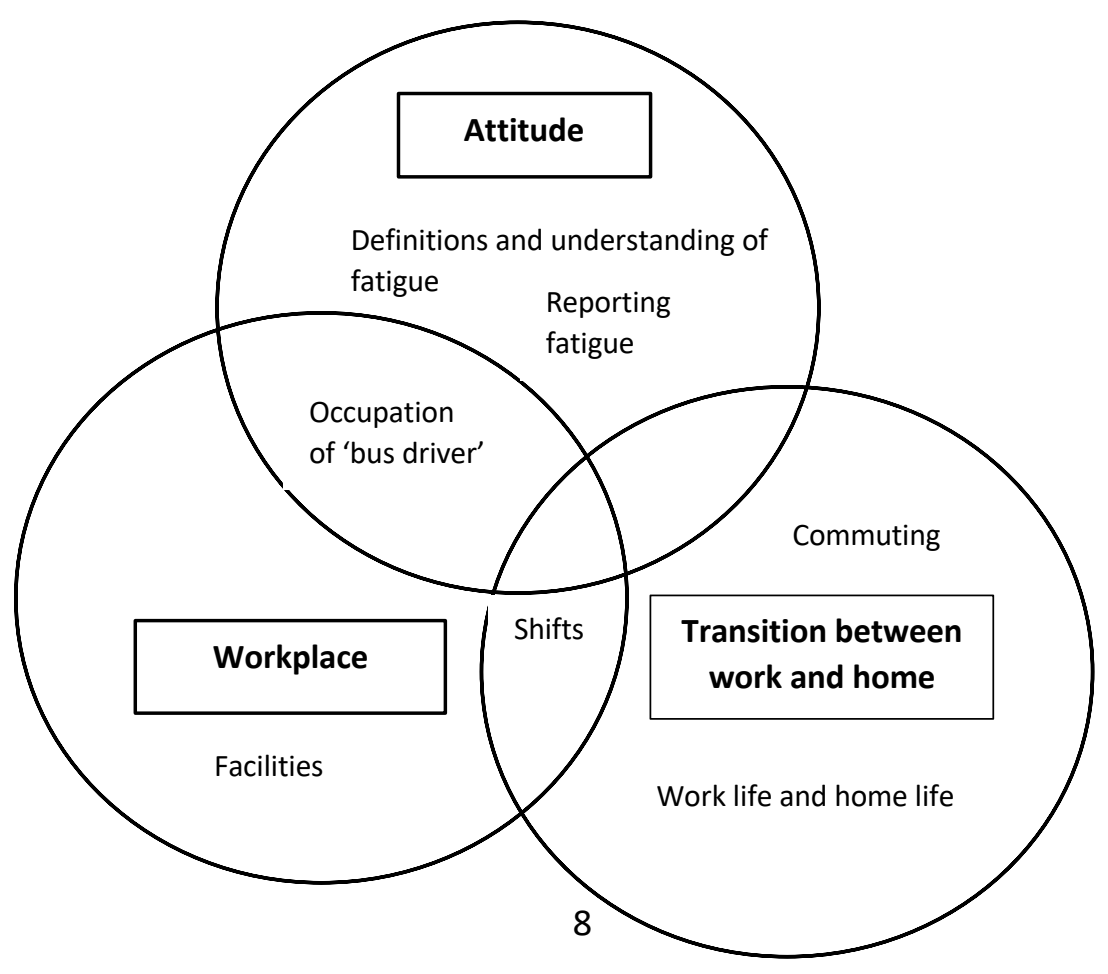


Figure 7: Pictorial representation of the focus group results

\subsection{Definitions and Understanding of Fatigue}

All of the participants (drivers and managers) acknowledged that they believe that fatigue is an issue amongst London bus drivers. One driver admitted: "I mean I'll be completely honest with you, I've fallen asleep behind the wheel of a bus before". A small number of participating drivers said they do not generally feel fatigued, but they recognise it amongst their colleagues, although one driver commented in relation to experiencing fatigue: "I'd have thought everybody, everybody. If they said they haven't, I think they'd be lying". Of the managers, all had seen examples of fatigue being a contributing factor to incidents on the road. The extent to which fatigue is considered a problem varied amongst the managers interviewed, but not all were as involved in investigating incidents and/or fatigue as others. Fatigue may be more often encountered by those closely involved in investigating accidents, thereby affecting their perceptions. Managers also commented on suffering from fatigue themselves, mostly due to working long hours or having lengthy commuting distances from home to work.

In order to reinforce the project definition of 'fatigue', participants were asked to discuss their understanding of the term at the beginning of each focus group and interview. In many cases, their first response was 'tired' or 'tiredness'. Lack of sleep, exhaustion, drowsiness, monotony caused by heavy traffic, time of a shift and insufficient rest time were also cited here, with drivers in particular being well aware that good quality sleep is the key to avoiding fatigue. The managers were more likely than the drivers to link tiredness explicitly with lack of concentration, crashes and falling asleep at the wheel. Drivers described fatigue as an impairment of ability and alertness due to the events of the day such as difficulties caused by passengers, traffic and radio/controllers. Other drivers mentioned boredom, irritability, being short tempered, losing concentration and slower reaction times.

\subsection{Reporting Fatigue}

There were differing views on reporting fatigue, with managers wishing to be open and approachable so that drivers can feel comfortable in talking to them about being tired. A good example of this is as follows:

"You know, I've asked questions, have they had enough rest, what's happening at home, you know, and that sort of stuff. And if they're prepared to talk to you they will. Sometimes they just bottle up and not want to talk. But if anybody says they're tired to me we've got - we have to act. Because touch wood nothing happens, if they go out and fall asleep behind the wheel it would come back to us. So we have to sort of be careful on what we do. It's not a case of just sort of getting the drivers and sending them out and not bothering with them, you know. You have some sort of relationship with the drivers so when you're on shift you get to know who's who. And if someone's not looking right you would ask questions if they were alright and 9 times out of 10 they will say they were okay ..."

Conversely, drivers generally would avoid reporting being fatigued, fearing disciplinary consequences. For example, in one focus group the following was said:

"Researcher: So when you say, they won't tolerate it, you mean you'd get in trouble for it? Respondent 4: Yeah, of course.

Respondent 1: Yeah, come to the office.

Researcher: What would they do?

Respondent 2: You'd get punished for it. 
Respondent 3: A written warning, final written warning, depends how many times you've done it."

The majority of drivers involved in the focus groups knew how to report fatigue, but many were unsure of how this would be handled by managers, and few said they have reported feeling fatigued. Notably, some drivers were comfortable with reporting fatigue, and had experience of doing so, citing their worries about the safety implications of carrying on working while tired. Others believed that they would face discipline if they admitted to feeling tired and suggested they would be more likely to call in sick in such a situation. In these cases there was generally a reluctance about being assessed by the operator, and potentially being offered medical support. Drivers suggested that they were 'not allowed' to be tired and were unlikely to discuss fatigue with managers, supervisors, or other drivers. This contrasts with the managers' emphasis on drivers to report fatigue so that they can receive help. Several managers said that in an incident which was found to be due to fatigue, a driver who had not reported fatigue would be more likely to be disciplined and sacked than one who had.

Individual differences exist between the participating managers in terms of how understanding they are about the fatigue which bus drivers exhibit, and how approachable they aspire to be. However, the managers all placed the emphasis and responsibility on the drivers to ensure that they are avoiding fatigue. They stated that companies are eager to support drivers in their efforts in this area but cannot force them to take the appropriate measures. Notably, all of the managers interviewed were positive about trying to help and support drivers if they report fatigue. They try to be approachable and open, desiring an open culture around tiredness issues, although all noted that the company as a whole could be more proactive in their efforts to highlight fatigue and promote a greater level of openness around the subject. Moreover, findings from the manager interviews support the finding of the focus groups that the drivers are reluctant to report fatigue due to fearing disciplinary action. They believe that drivers will either continue working although fatigued, or report in sick rather than admit to fatigue.

The managers also discussed the difficulty of identifying fatigue as a contributory factor in an incident. In all companies there are investigations of incidents, with procedures and questions asked, including some related to sleep, shift patterns, overtime and fatigue. In addition, many of the managers were responsible for watching recordings of drivers in incidents. Some had gained a knowledge of the signs of potential fatigue but noted it can be impractical to prove that it has been in play.

\subsection{Shift Patterns and Overtime}

A wide variety of shift patterns and overtime was being offered by the operating companies and therefore undertaken by the drivers, the specifics of which will not be discussed here. However, there follows a consideration of the key issues which were debated. Importantly, there is agreement across the two work roles that shift patterns and overtime are a key contributor to fatigue.

Shift work was cited by drivers as causing fatigue and was described as affecting the body's systems due to severe changes in start and end times: "one day you might start six, the next day you start seven, and then the next day you start four, so you haven't got a proper body clock schedule". This may be due to working a mixed rotating shift rota (from early to middle to late) or having a variability between start times when on the same type of shift. Sleep deprivation was a particular concern, with suggestions that drivers find it difficult to sleep at unconventional times (e.g. 19:00) or may be out late socialising and so will be having a limited amount of effective sleep in advance of shifts beginning. 
With regard to rotas, the focus groups showed that drivers believe they are a significant issue in relation to fatigue. Drivers commented on the difficulties of their shifts changing from week to week. For example, on a regular rota one week might be early shifts, the next middles and the next late shifts. Some of the drivers noted that their body clock becomes used to a certain sleep pattern for one week and then quickly has to change sleep pattern - they find it hard to adjust. This then affects eating (maybe having lunch or breakfast at 10:00). The next week the shift might begin at 16:00 and lunch will be at 19:00 or 20:00, necessitating a change in body clock every week. Having a week with very early morning shifts was said to cause cumulative sleep deprivation and fatigue, as were sudden shift changes.

The drivers were all knowledgeable about the legal limits of driving, and most companies have a driver portal or other software to inform drivers of their shifts and allow them to check their hours are compliant. This was very important when swapping shifts; the arrangements were routinely left to drivers to decide between themselves but then checked by a scheduler or manager before being made official: "We've all got fixed shift pattern but it's your choice if you want to change your job then someone's willing to change then you can change them ... As long as you've got someone you can change with". All operating companies allow drivers to swap shifts within the legal limits. This was seen as a potential contributor to fatigue by both the drivers and the managers.

With all participants agreeing about the effect of shift patterns, the managers who were interviewed were generally apt to be flexible on this subject, suggesting patterns might be amended and that their employers are instigating measures aimed at improving fatigue management. One manager noted that they believe the operator's safety and scheduling teams are not working together to the extent they could. This might aid in the creation of safer shift patterns. There was also a general wish from drivers for shorter hours with better pay, which it was thought would lead to a reduction in fatigue.

Overrunning shifts were cited during the driver focus groups as a particular contributor to fatigue, where a driver might be delayed significantly due to traffic and/or diversions. Some focus group participants also saw an increasing pressure to arrive early to work (often unpaid) in order to check the bus before departure, demonstrating a need to maintain buses to ensure they are always kept in a suitable condition. As stated by one driver: "I come to work and I think, "Will it be a good bus or bad bus?" If I get a bad bus in the morning, then I know I will be late all the time. I will have no stand time and so on. Maybe I will be lucky, I will have a bus that actually drives, the doors open and close and no problems".

Managers and drivers agreed that, as an integral part of shift patterns, overtime contributes to levels of fatigue. Drivers were well-informed about how overtime fits in with their other driving duties; within the legal limits it is possible to drive 13 days out of 14, of which some of the participants have experience. This necessarily cuts down on rest periods; some drivers commented on these not being long enough in terms of sleeping sufficiently well.

Both groups had their own reasons for seeing a necessity for overtime. The managers all discussed having a shortage of drivers, leading them to offer relatively high levels of overtime to existing employees. They generally realise this could be leading to drivers taking on too much work, but their responsibility to the operator and knowledge of a driver's own wishes outweigh those concerns. Another motivator for overtime cited by both the drivers and the managers was the low levels of pay that drivers experience. They see this as a result of the tendering process in which companies endeavour to keep costs as low as possible. Drivers will therefore take on extra duties to make up their money. 


\subsection{Work and Home Life}

Drivers discussed the difficulties of balancing their work and home life, and how this can lead to feeling tired. For example, drivers interested in overtime can be contacted by their employer whilst at home and this can often interrupt them whilst they are actually asleep; this is not ideal for effective sleep patterns.

Many of the participants discussed family and social commitments as important causes of fatigue. They noted that their non-working time is often taken up with the needs of their families, causing them to miss out on rest, relaxation and sleep time: " ... you can leave work, you're exhausted and then you're going home, and you're going home to family life, and it's not always just simply - oh hi babe, your dinner's on the table. You don't know what's happening with your family, your kids, i.e. you need to spend time, so it's a whole other stress".

It is particularly striking that drivers may have only a 10-hour period between shifts, during which they must, for example, travel between work and home, cook and eat some food, de-stress and relax, spend time with their family and, most importantly, sleep. Many participants noted finding this problematic. This was also true, particularly for younger drivers, when considering social commitments which might cause drivers to stay up late, therefore lacking sufficient sleep for a subsequent shift.

The managers recognise drivers have a life outside of work and noted that family and other commitments are likely to affect their ability to have sufficient sleep and are therefore a contributory cause in levels of fatigue. They also acknowledge that the younger drivers may have social commitments which can reduce their levels of sleep.

\subsection{Commuting}

The focus groups showed that commuting can be a significant contributor to fatigue. Drivers might live a long distance from the garage or depot at the start of their route, with drivers and managers commenting that their salary is insufficient to live in central London. Reported commuting times of up to one and a half to two hours each way were quoted by participants; either for themselves or for colleagues. For example: "You've got to work out that not everyone lives round the corner to the garage, it takes me an hour and a half to get to work, an hour and a half to get home. So you take that, if I've got ten hours off, three hours travelling in that." One influencing factor on commute times is the tendering process for bus routes. A driver's home may remain the same but commuting times can change as a result of a certain route passing between operating companies resulting in a change in work location for any particular driver. These long periods of time spent travelling to work are thought by drivers to be a contributor to fatigue, as they may informally add to the hours a driver spends on the road. Any long commuting times can impact on rest and social and family time, potentially having an effect on fatigue. It is clear, however, that commuting by public transport does permit drivers to sleep before beginning a shift; several said that they take advantage of this possibility if they can.

Some routes do not begin at a garage or depot, rather the start point is at a bus stop (or a different garage or depot from where drivers sign in to work) and this requires drivers to travel (usually by public transport) after starting work but before they begin bus driving. This is included in the work time and is paid, however, it can be a cause of additional pressure and fatigue to drivers due to trains and/or buses running late. Some drivers said they feel it necessary to arrive at work disproportionately early (before the time for which they are paid) to ensure a timely arrival to the location where they pick up their bus. 
The managers claimed that none of the operating companies have a policy or restriction on commuting distance to work, but they were all aware that there are drivers who have a long journey before they sign on for duty. They are conscious that this can add to fatigue and some mentioned either speaking to employees about this or monitoring those to whom it applies: "Because we have quite a few people living out in Essex, you see, so their commute would be about ... an hour, or just under an hour. I don't think we've actually kept a close eye on it, but there was just one guy who we knew it was two hours, and that kind of like set alarm bells ringing for us".

\subsection{Facilities}

A lack of suitable facilities was often cited by drivers as a contributor to fatigue as they impact on ability to rest and recover from driving. Canteen facilities are provided at many garages and depots but are likely to have limited opening times and will not necessarily be open during early or late shifts. Furthermore, participants claimed that the canteens they do have are unlikely to offer healthy food. In other places, microwave ovens are available, but often shared amongst a large number of people making it difficult to gain access during a standard break. Where there is no canteen at a changing point it is often necessary for drivers to commute to a place to find food, and eat a heavy meal causing feelings of drowsiness when returning to drive again.

A general lack of rooms in which to rest at the garages and depots was also cited as a barrier to being able to relax and perhaps sleep before shifts and during breaks (including those which are part of a spread-over shift). Participants clearly believe this to impact on fatigue levels. Drivers said they often do not have access to toilet facilities when on the road, causing them not to drink sufficient liquid for fear of needing to urinate.

Drivers are aware of the positive effects of exercise, both for general health and in helping to avoid fatigue. To this end, they would like more of their employers to provide suitable exercise equipment, shower facilities or discounted access to a local gym.

\subsection{The Occupation of 'Bus Driver'}

The bus drivers saw their role as a somewhat distinctive occupation. In particular, the notion that they hold a high level of responsibility was universally understood, with drivers noting their heightened feelings of accountability for other people's safety whilst driving a heavy vehicle full of passengers: "We have a responsibility and we've got passenger safety at hand". There was also a constant feeling of being responsible for delivering a vital service to the public which drivers want to maintain without being subject to delay.

The drivers acknowledged a difference in their driving when fatigued and noted that, in their opinion, the key effects were: speeding up, driving erratically, driving on autopilot, slower reaction times, failure to think and plan ahead, losing concentration, and driving aggressively. The manager interviewees had observed these when considering fatigue either in a general sense, or in specific incidents. Drivers were aware of the possibility of making a simple mistake and causing an incident due to fatigue. A clear link between fatigue and accidents was recognised by both drivers and managers.

It was clear that the first concern of the managers interviewed is safety and they are in some ways caught in the middle between drivers and the needs of the operator because there is a requirement to keep the buses moving at the same time as guarding the operator's reputation. From the management perspective it is necessary for supervisors and managers to know how to spot the signs of fatigue, question it and challenge it and make sure the people who are driving their buses are fit 
to do so. As noted by one manager: "we often say to drivers, oh you have to make sure that you're fit to be at work; and often drivers come into us and say, well this is how I'm feeling, do you think I should drive, and my answer to anyone who says that to me, is that unfortunately I don't know how you're actually feeling, so you need to make the decision on whether or not you feel it's safe for you to be driving, and if not, then you obviously need to not be driving at the moment".

On the other side managers stated that there is a need to make sure the staff are aware of fatigue issues and that they are actively managing it themselves taking into account their sleep patterns and what they do during the day and what they do before and after.

\section{Discussion}

The qualitative approach (focus groups and interviews) employed in this research has permitted an investigation of the personal experiences and opinions relating to fatigue of London bus drivers and their managers. It is the first time such a detailed exploration of this kind has been undertaken.

The research found that fatigue is both existent and a concern for London bus drivers; participants in the focus groups and interviews are agreed on this point, which concurs with the findings of Vennelle et al. (2010), Anund et al. (2016), Deza-Becerra et al. (2017), Kim et al. (2018) and Santos et al. (2013). The presence of fatigue was also evident in Biggs et al. (2009), the main other qualitative study in this area. Fatigue is a problem amongst participants in the focus groups, with all of the participants having experience of sleep-related incidents whether their own or a colleague's. In common with findings from Vennelle et al. (2010), the managers had all seen examples of this. This 2009 study shows that, ten years on from Biggs et al. (2009), views on fatigue are generally shared by drivers in the current research and have therefore not altered greatly in the intervening years.

When asked to define fatigue, many of the drivers and managers noted believing that lack of sleep, poor sleep quality and tiredness are key issues. Indeed, research has shown that one of the main causes of fatigue related incidents are attentional lapses due to insufficient sleep (Philip and Åkerstedt, 2006; Philip et al., 2005; Schwarz et al., 2016; Chaiard et al., 2019).

When the results of the two tasks are compared, it is evident that the managers who were interviewed are more apt to understand the perspectives of the participating drivers than the drivers are to be understanding about the views of the managers. This is perhaps not surprising, with drivers seeing the managers as the individuals who organise and can discipline them and the majority of the managers having previously worked as drivers; this gives them direct experience of the role of driver. This finding is related to the idea that the responsibility for managing fatigue lies both with the organisation and the individual (Gander et al., 2011; Anund et al., 2016), and that working together would aid mutual understanding at a higher level. This is also reflected in Biggs et al. (2009) in which drivers reported that "managerial support was important ... in predicting the likelihood that a driver would feel comfortable taking steps to prevent driving fatigue" (p. 2).

The drivers in the focus groups claimed that they do not discuss fatigue amongst themselves, or with their managers and generally avoid reporting it, fearing disciplinary consequences. This was found by Miyama et al. (2020) in bus drivers but it is not a unique problem for bus drivers and was detected amongst train drivers in Australia and New Zealand by Filtness and Naweed (2017). Conversely, many of the managers interviewed demonstrate a wish to make fatigue a more acceptable subject to discuss and would welcome a more open culture around this subject. The managers are making attempts to mitigate against fatigue by responding to the needs of the drivers when fatigue is reported to them. However, the managers all stated that the responsibility lies with the drivers to manage and report their own fatigue. These findings reflect the effect that managerial support can 
have on fatigue levels, as found by Biggs et al. (2006 and 2009), in which depots that reported positive relationships with management reported less fatigue than those that reported negative relationships (p. 2), and the need for a holistic approach to prevent fatigue-related crashes (Mohamed et al., 2012). In order to mitigate this, an open culture in terms of fatigue could be established, moving away from a discipline-based culture so that individuals feel supported. Furthermore, the promotion of a working environment which encourages drivers to voluntarily report health problems was found in a survey of Japanese bus drivers (Miyama et al., 2020), together with a need for more comfortable well-equipped rest facilities. It is likely that everyone will experience aspects of fatigue at some point, whether through scheduling, poor sleep, or family/social circumstances, so it is important that a management system is in place. Indeed, Miyama et al. (2020) found that continuing driving when feeling sick was a major risk factor for collisions and near-miss incidents.

Shift patterns are agreed by the participating London bus drivers and managers to be a key issue in the presence of fatigue; this includes rota patterns, scheduling and break length. There is an understanding that these might therefore be improved, and that safety and scheduling teams could work together more effectively. Other research has similarly identified reports of tight or unrealistic route schedules, shift irregularity and extended shift cycles as contributors to fatigue (see, for example, Wang \& Wu, 2019; Abdullah \& Von, 2011; Biggs et al., 2006; Miyama et al., 2020). The other qualitative study in this area featuring focus groups with bus drivers identified tight route schedules and a corresponding restriction on shift breaks as significant factors affecting fatigue levels, together with adverse turn-around periods between shifts and irregularity of shifts (Biggs et al., 2009).

The research confirms that both drivers and managers believe shift schedules directly impact ability to rest, sleep, and achieve a good work/life balance; a finding which was in evidence in Miyama et al.'s survey (2020). In this research the managers discussed a range of options which they offer when drivers report feeling fatigued. The research also shows that there are many opportunities for overtime for London bus drivers who suggested that these opportunities are taken up by many workers as a response to what they consider to be low levels of pay. Both the managers and the drivers agreed that overtime contributes to levels of fatigue, since it adds to the number of total hours being worked by individuals. With research having found that cumulative fatigue increases with the number of days and hours worked (Sando et al., 2010) and that multi-day driving patterns are associated with higher crash risk (Wang \& Wu, 2019), high levels of overtime are clearly a concern. This is reflected in the qualitative study by Biggs et al. (2009) which identified extended shift cycles (12-days on, 2-days off) as a significant factor in fatigue and in which it was generally agreed that drivers working these shift patterns are doing so for economic reasons.

The responsibility inherent in the job of bus driver was recognised by both the participating drivers and managers. There was also a feeling of being responsible for delivering a vital service to the public. The participating managers also demonstrated that their first concern is safety and they were therefore more likely than the drivers to link fatigue/tiredness explicitly with lack of concentration, crashes and falling asleep at the wheel. This reflects the wealth of research which has linked fatigue to safety issues (see Williamson et al., 2011 for review). Managers have a responsibility both to the drivers and to the needs of the company; to the managers, fatigue is a safety issue and they feel the responsibility to make sure it is not having an adverse impact on the business or risking people's lives.

As with all research the findings should be considered in the context of the limitations. It should firstly be noted that the research was concentrated around London, the transport systems of which 
could be seen as a particular and unusual circumstance. As a result, the participants were all metropolitan drivers within just one city. A second limitation is that, due to operational limitations, it was not possible to hold consistent focus groups across all ten of the relevant operators. However, representatives of all of the operators successfully took part in the research with eight of the ten groups being relatively uniform in nature.

Another limitation of the current research related to the participants in that one group which was not widely investigated were night drivers. Although some occasional night drivers participated in the focus groups, in most cases night drivers only work nights, and would therefore have been unavailable to take part in our discussion groups since these occurred during the day.

\section{Conclusions}

It is concluded from the research that fatigue is existent and a concern for London bus drivers and their managers. As might be expected, there are differences in perception about fatigue between the bus drivers and managers. One of the main differences relates to reporting fatigue, with managers claiming they would welcome drivers reporting fatigue, but drivers not feeling comfortable reporting fatigue for fear of disciplinary actions. This will necessarily make it difficult to establish the extent of fatigue in the industry, and thereby to attempt to alleviate it.

An important conclusion is that an open culture where drivers feel able to discuss fatigue would be beneficial to all and is currently sought by many of the managers who were interviewed. The connection between sleep and fatigue is also clearly understood by all the participants. Both bus drivers and managers in the study recognise the responsibility they hold for the safety of their passengers and can relate this to issues surrounding fatigue.

\section{Acknowledgements}

The authors gratefully acknowledge the contribution to this research of the participants and Transport for London (TfL) for their funding and support.

Also gratefully acknowledged are Anna Anund, Anna Sjörs Dahlman and Jonas Ihlström from the Swedish National Road and Transport Research Institute (Linköping, Sweden) who were the collaboration partners for this research.

\section{References}

Abdullah, D. N. M. A., \& Von, H. L. (2011). Factors of fatigue and bus accident. In Proceedings of the 2nd International Conference on Innovation, Management and Service: International Proceedings of Economics Development and Research (Vol. 14, pp. 317-322).

Anund, A., Ihlström, J., Fors, C., Kecklund, G., \& Filtness, A. (2016). Factors associated with selfreported driver sleepiness and incidents in city bus drivers. Industrial health, 54(4), 337-346. DOI: 10.2486/indhealth.2015-0217

Biggs, H. C., Dingsdag, D. P., \& Stenson, N. J. (2006). Fatigue issues for metropolitan bus drivers: Ramifications of quantitative and qualitative research findings for safety management. Paper presented at the 2006 Australasian Road Safety Research, Policing and Education Conference. Surfers Paradise, Queensland. 
Biggs, H. C., Dingsdag, D. P., \& Stenson, N. J. (2009). Fatigue factors affecting metropolitan bus drivers: a qualitative investigation. A Journal of Prevention, Assessment and Rehabilitation, 32(1), 510. DOI: $10.3233 /$ WOR-2009-0810

Bioulac, S., Franchi, J. A. M., Arnaud, M., Sagaspe, P., Moore, N., Salvo, F., \& Philip, P. (2017). Risk of motor vehicle accidents related to sleepiness at the wheel: a systematic review and metaanalysis. Sleep, 40(10). DOI: 10.1093/sleep/zsx134

Braun, V. \& Clarke, V. (2006) Using thematic analysis in psychology, Qualitative Research in Psychology, 3(2), 77-101. DOI: 10.1191/1478088706qp063oa

Chaiard, J., Deeluea, J., Suksatit, B., \& Songkham, W. (2019). Factors associated with sleep quality of Thai intercity bus drivers. Industrial Health, 2018-0168. DOI: 10.2486/indhealth.2018-0168

Connor, J., Norton, R., Ameratunga, S., Robinson, E., Civil, I., Dunn, R., \& Jackson, R. (2002). Driver sleepiness and risk of serious injury to car occupants: population-based case control study. Bmj, 324(7346), 1125. DOI: 10.1136/bmj.324.7346.1125

Deza-Becerra, F., de Castro, J. R., Gonzales-Gonzales, C., León-Jiménez, F. E., Osada-Liy, J., \& RosalesMayor, E. (2017). Sleep habits, fatigue, and sleepiness in Chiclayo-Peru's bus drivers. Sleep and Breathing, 21(3), 745-749. DOI: 10.1007/s11325-017-1502-9.

Filtness, A. \& Naweed, A. (2017). Causes, consequences and countermeasures to driver fatigue in the rail industry: The train driver perspective. Applied Ergonomics, 60, 12-21. DOI:

10.1016/j.apergo.2016.10.009

Gander, P., Hartley, L., Powell, D., Cabon, P., Hitchcock, E., Mills, A., \& Popkin, S. (2011). Fatigue risk management: Organizational factors at the regulatory and industry/company level. Accident Analysis \& Prevention, 43(2), 573-590. DOI: 10.1016/j.aap.2009.11.007

Horne, J. A., \& Reyner, L. A. (1995). Sleep related vehicle accidents. Bmj, 310(6979), 565-567. DOI: 10.1016/S1369-8478(01)00014-6

Kim, S. M., Um, Y. H., Kim, T. W., Jeong, J. H., Seo, H. J., Song, J. H., \& Hong, S. C. (2017). Excessive Daytime Sleepiness and Its Risk Factors for Commercial Bus Drivers in Korea. Sleep Medicine Research, 8(2), 76-80. DOI: 10.17241/smr.2017.00122

Miller, K. A., Filtness, A.J., Anund, A., Maynard, S. \& Pilkington-Cheney, F. (2020) Contributory factors to sleepiness amongst London bus drivers. Transportation Research Part F, 78, 415-424. DOI: 10.1016/j.trf.2020.07.012

Miyama, G., Fukumoto, M., Kamegaya, R. \& Hitosugi, M. (2020) Risk Factors for Collisions and NearMiss Incidents Caused by Drowsy Bus Drivers. International Journal of Environmental Research and Public Health, 17(12), 4370. DOI: 10.3390/ijerph17124370

Mohamed, N., Mohd-Yusoff, M. F., Othman, I., Zulkipli, Z. H., Osman, M. R., \& Voon, W. S. (2012). Fatigue-related crashes involving express buses in Malaysia: Will the proposed policy of banning the early-hour operation reduce fatigue-related crashes and benefit overall road safety? Accident Analysis \& Prevention, 45, 45-49. DOI: 10.1016/j.aap.2011.09.025

Philip, P., \& Åkerstedt, T. (2006). Transport and industrial safety, how are they affected by sleepiness and sleep restriction? Sleep medicine reviews, 10(5), 347-356. DOI: 10.1016/j.smrv.2006.04.002 
Philip, P., Sagaspe, P., Moore, N., Taillard, J., Charles, A., Guilleminault, C., \& Bioulac, B. (2005). Fatigue, sleep restriction and driving performance. Accident Analysis \& Prevention, 37(3), 473-478. DOI: 10.1016/j.aap.2004.07.007

Philip, P., Chaufton, C., Orriols, L., Lagarde, E., Amoros, E., Laumon, B., \& Sagaspe, P. (2014). Complaints of poor sleep and risk of traffic accidents: a population-based case-control study. PloS one, 9(12), e114102. DOI: 10.1371/journal.pone.0114102

Pilkington-Cheney, F., Filtness, A. J., \& Haslam, C. (2020a). A qualitative study exploring how city bus drivers manage sleepiness and fatigue. Chronobiology International, 1-11. DOI:

$10.1080 / 07420528.2020 .1812623$

Pilkington-Cheney, F., Filtness, A., Jones, W., Maynard, S., Gibb, A. \& Haslam, R. (2020b)

Biomathematical modelling for managing worker fatigue in civil engineering. Proceedings of the Institution of Civil Engineers - Civil Engineering. DOI: 10.1680/jcien.20.00009.

Saldana, J. (2009) An introduction to codes and coding. The Coding Manual for Qualitative Researchers. London, UK: Sage.

Sando, T., Mtoi, E., \& Moses, R. (2010). Potential Causes of Driver Fatigue: A Study on Transit Bus Operators in Florida. In Transportation Research Board of the National Academies' 2011 90th Annual Meeting, paper (No. 11-3398).

Santos, D. B., Bittencourt, L. G., de Assis Viegas, C. A., \& Gaio, E. (2013). Daytime sleepiness and attention in city bus drivers of two capitals of Brazil. Revista Portuguesa de Pneumologia (English Edition), 19(4), 152-156. DOI: 10.1016/j.rppnen.2013.06.003

Schwarz, J. F., Geisler, P., Hajak, G., Zulley, J., Rupprecht, R., Wetter, T. C., \& Popp, R. F. (2016). The effect of partial sleep deprivation on computer-based measures of fitness to drive. Sleep and Breathing, 20(1), 285-292. DOI: 10.1007/s11325-015-1220-0

Transport for London, 2019 https://tfl.gov.uk/corporate/about-tfl/what-we-do/buses (accessed 30.04.19)

Vehicle Operator Services Agency [V. O. S. A] (2009) Rules on Drivers' Hours and Tachographs: Passenger-Carrying Vehicles in the UK and Europe. London, UK: Driver and Vehicle Standards Agency.

Vennelle, M., Engleman, H. M., \& Douglas, N. J. (2010). Sleepiness and sleep-related accidents in commercial bus drivers. Sleep and breathing, 14(1), 39-42. DOI: 10.1007/s11325-009-0277-z

Wang, S. Y., \& Wu, K. F. (2019). Reducing intercity bus crashes through driver rescheduling. Accident Analysis \& Prevention, 122, 25-35. DOI: 10.1016/j.aap.2018.09.019

Williamson, A., Lombardi, D. A., Folkard, S., Stutts, J., Courtney, T. K., \& Connor, J. L. (2011). The link between fatigue and safety. Accident Analysis and Prevention, 43(2), 498-515. DOI: 10.1016/j.aap.2009.11.011

Zhang, H., Yan, X., Wu, C., \& Qiu, T. (2014). Effect of circadian rhythms and driving duration on fatigue level and driving performance of professional drivers. Transportation Research Record: Journal of the Transportation Research Board, (2402), 19-27. DOI: 10.3141/2402-03 